\title{
KLAUS-DieTER DRÜEN
}

\section{Der Weg zur gemeinnützigen (rechtsfähigen) Stiftung - Stiftungszivilrechtliche Gestaltungsmöglichkeiten und steuerrechtliche Vorgaben}

Der Stiftungsboom in Deutschland ist ungebrochen. Während die Zahl rechtsfähiger Stiftungen im Jahre 1980 noch bei 880 lag und jährlich ungefähr 120 Stiftungen gegründet wurden, ist sie im Jahr 2008 auf 16.406 angewachsen. Allein im Jahre 2008 wurden 1.020 neue Stiftungen gegründet. ${ }^{1}$ Die Mehrzahl der Stiftungen ist dabei gemeinnützig. Nach Schätzungen soll dies für 95 Prozent aller Stiftungen gelten. ${ }^{2}$ Der Staat fördert Gemeinwohl fördernde, private Organisationen durch eine Steuerfreistellung bei den Ertragsteuern. Verfassungsrechtlich rechtfertigt sich diese Förderung für die das Gemeinwohl fördernden Organisationen durch die Prinzipien der Subsidiarität und der Staatssubstitution. ${ }^{3}$ Stiftungen sind taugliche Gemeinnützigkeitssubjekte. ${ }^{4}$ Verfolgt eine Stiftung nach Satzung und tatsächlicher Geschäftsführung ausschließlich und unmittelbar gemeinnützige, mildtätige oder kirchliche Zwecke (steuerbegünstigte Zwecke), genießt sie nicht nur selbst eine Steuerverschonung, sondern der Staat prämiert zugleich den Stifter durch den ertragsteuerlichen Zuwendungsabzug. Denn die Prinzipien der Subsidiarität und der Staatssubstitution rechtfertigen nicht nur die Steuerentlastung der zur Förderung des Gemeinwohls tätigen Organisation selbst, sondern sie erstrecken sich auch auf die Personen, die diese mit finanziellen Mitteln versorgen. ${ }^{5}$ Dabei hat der Staat unlängst die steuerlichen Anreize für Stifter durch das „Gesetz zur weiteren Stärkung des bürgerschaftlichen Engagements“ ausgedehnt ${ }^{6}$ und dabei vor allem die speziellen Abzugsmöglichkeiten bei (Zu-)Stiftungen intensiviert. ${ }^{7}$ Gerade wegen der besonderen Förderanreize für das Stiftungsengagement stellt sich für viele Stiftungswillige die Frage nach dem ,richtigen Weg zur guten Tat““.

\section{Grundzüge des Stiftungszivilrechts}

Eine Stiftung im Sinne der $\S \S 80$ ff. BGB ist eine mit Rechtsfähigkeit ausgestattete nicht verbandsmäßig organisierte Einrichtung, die einen vom Stifter bestimmten Zweck mit Hil-

1 Quelle: Bundesverband deutscher Stiftungen, http://www.stiftungen.org (30.04.2009).

2 Richter $\left({ }^{3}\right.$ 2009: $\$ 98$ Rn. Vor 1).

3 Näher Isensee (2003: 96 ff.) und Seer (2003: 21 ff.); zusammenfassend Drüen (2008: 445).

4 Zum Kreis der steuerbegünstigten Körperschaften im Einzelnen Hüttemann (2008: § 2).

5 Seer (2003: 26).

6 Gesetz vom 10.10.2007, BGB1. I 2007, 2332. Dazu näher der Beitrag von Förster in diesem Jahrbuch.

7 Nach § 10b Abs. 1a EStG n.F. können Spenden in den Vermögensstock öffentlich-rechtlicher und gemeinnütziger Stiftungen auf Antrag des Steuerpflichtigen in einem Zehnjahreszeitraum ab der Zuwendung bis zu einem Gesamtbetrag von einer Million € (zuvor $307.000 €$ ) zusätzlich zu den Höchstbeträgen nach $\S 10 b$ Abs. 1 Satz 1 EStG abgezogen werden. Überdies unterfallen seit dem Jahr 2007 auch bloße Zustiftungen, durch die keine neue (unselbständige) Stiftung gegründet wird, als Spenden in den Vermögensstock dem erhöhten Spendenabzug nach $§ 10$ Abs. 1a EStG. Zur Anwendung vgl. BMF v. 18.12.2008, BStB1. 2009 I, 16. 
fe eines dazu gewidmeten Vermögens dauerhaft fördern soll. ${ }^{8}$ Im Zeitpunkt ihrer Errichtung erlangt die Stiftung eine rechtliche Unabhängigkeit vom Stifter. Im Hinblick auf die Ausgestaltung der Stiftung, was den Förderzweck, die Organisation und ihre Vermögensausstattung betrifft, hat er zwar einen großen Entscheidungsraum, doch sobald die Stiftung ihre Rechtsfähigkeit erlangt hat, ist seine Einflussnahme äußerst begrenzt. ${ }^{9}$ Eine Stiftung ist, wenn man so will, eine „Einbahnstraße“.

Aus verschiedenen Gründen konzentriert sich die weitere Darstellung auf die lebzeitige Einrichtung einer rechtsfähigen Stiftung. Eine rechtsfähige Stiftung kann zwar auch von Todes wegen errichtet werden; sie entsteht mit dem Todestag des Stifters, vorbehaltlich der späteren Anerkennung durch die Stiftungsbehörde (vgl. § 84 BGB). ${ }^{10}$ Für die lebzeitige Stiftung sprechen aber mehrere Gründe: Zunächst bietet sie für den Stifter den Vorteil, dass er die Gründung selbst und den Aufbau der Stiftungsarbeit noch tatkräftig begleiten kann. ${ }^{11}$ Aus steuerrechtlicher Sicht ist sie vorzugswürdig, weil sie dem Stifter den Sonderausgabenabzug für die Erstkapitalausstattung nach $\S 10 \mathrm{~b}$ EStG ermöglicht. ${ }^{12}$

Wesentliche Merkmale einer zu Lebzeiten des Stifters errichteten rechtsfähigen Stiftung sind nach $\S \S 80 \mathrm{ff}$. BGB der Stiftungszweck, das Stiftungsvermögen und die Stiftungsorganisation. Dazu bestimmt $\S 81$ Abs. 1 BGB:

Das Stiftungsgeschäft unter Lebenden bedarf der schriftlichen Form. Es muss die verbindliche Erklärung des Stifters enthalten, ein Vermögen zur Erfüllung eines von ihm vorgegebenen Zweckes zu widmen. Durch das Stiftungsgeschäft muss die Stiftung eine Satzung erhalten mit Regelungen über:

den Namen der Stiftung,

den Sitz der Stiftung,

den Zweck der Stiftung,

das Vermögen der Stiftung,

die Bildung des Vorstands der Stiftung.

Die Satzung ist das Kernstück der Verfassung der Stiftung. ${ }^{13}$ Die Verfassung der Stiftung wird durch das Stiftungsgeschäft ( $\$ 81$ BGB), aber vor allem durch die Satzung bestimmt ( $\$ 85$ BGB). Das Stiftungsgeschäft unter Lebenden ist ein einseitiges, nicht empfangsbedürftiges Rechtsgeschäft. ${ }^{14}$ Es ist bedingungsfeindlich. Auch wenn das Stiftungsgeschäft die Einbringung von Grundstücken in die Stiftung vorsieht, genügt nach überwiegender Ansicht die Schriftform und es ist keine notarielle Beurkundung erforderlich, zumal das Anerkennungsverfahren dieselbe Richtigkeitsgewähr und Warnfunktion bietet. $^{15}$

\footnotetext{
8 Heinrichs/Ellenberger ( ${ }^{68}$ 2009: Vorb v § 80 Rn. 5); Schlüter/Stolte (2007: 31); Werner (2006: 539).

9 Schlüter/Stolte (2007: 31).

${ }^{10}$ Näher Bianchini-Hartmann/Richter (2008: 340 f.); Hüttemann (2008: § 2 Rn. 55).

${ }^{11}$ Ebenso Hüttemann (2008: $\$ 2$ Rn. 56).

${ }^{12}$ Heinicke ( ${ }^{28}$ 2009: $§ 10 \mathrm{~b}$ Rn. 70 ff.).

${ }^{13}$ Heinrichs/Ellenberger ( ${ }^{68} 2009$ : $§ 81$ Rn. 5).

${ }^{14}$ Heinrichs/Ellenberger ( ${ }^{68} 2009$ : $§ 81$ Rn. 2); Werner ( ${ }^{12} 2008$ : $\S 81$ Rn. 3).

${ }^{15}$ Werner ( ${ }^{12} 2008$ : $\S 81$ Rn. 4); anderer Ansicht Heinrichs/Ellenberger ( ${ }^{68} 2009$ : $\S 81$ Rn. 3) und Troll/Wallenhorst/Halaczinsky ( ${ }^{5}$ 2004: A Rn. 38).
} 
Der Stiftungszweck wird vom Stifter im Stiftungsgeschäft festgesetzt und muss auf Dauer angelegt sein. Er kann gemeinnützig oder privatnützig sein. ${ }^{16}$ Rein privatnützliche Ziele schließen freilich eine steuerrechtliche Begünstigung aus.

Das Stiftungsvermögen ist gleichfalls ein konstitutives Merkmal der Stiftung. Zu den gesetzlichen Voraussetzungen gehört neben der Satzung mit den gesetzlich geforderten Mindestangaben ein für die nachhaltige Zweckerreichung ausreichendes Vermögen. Als Stiftungsvermögen kommen in erster Linie Geld, Wertpapiere einschließlich Aktien, Anteile an Kapitalgesellschaften, Beteiligungen an Personengesellschaften, stille Beteiligungen, Grundstücke und andere Vermögensgegenstände in Betracht. ${ }^{17}$ Die Werte, die der Stiftung mit der Maßgabe zugewandt werden, dass sie nicht verbraucht werden, sondern dauerhaft in der Stiftung verbleiben, bilden das Grundstockvermögen als das materielle Fundament der Stiftung. Aus ihm erzielt die Stiftung Erträge. Soweit die Satzung nichts anderes vorsieht, soll die Stiftungstätigkeit grundsätzlich nur aus den Erträgen finanziert werden. In diesem Fall gilt der später noch anzusprechende Grundsatz der Vermögenserhaltung. ${ }^{18}$ Weder das BGB noch die Landesstiftungsgesetze schreiben ein Mindestvermögen für die Errichtung der Stiftung vor. Da aber die dauerhafte und nachhaltige Erfüllung des Stiftungszwecks gesichert sein muss, gehen die Stiftungsbehörden im Allgemeinen davon aus, dass ein Ertrag bringendes Stiftungsvermögen von mindestens $50.000 €$ vorhanden sein muss. ${ }^{19}$ Dafür bedarf es einer Prognoseentscheidung. Nach der behördlichen Anerkennung müssen die einzelnen Gegenstände vom Stifter der Stiftung übereignet werden. Forderungs-, Mitgliedschafts- und andere abtretbare Rechte gehen nach $\S 82$ Satz 2 BGB automatisch im Wege der Legalzession mit der Anerkennung der Stiftung über. ${ }^{20}$

Die Stiftung unterscheidet sich in ihrer Organisation durch ihre nicht verbandsmäßige Struktur von den anderen juristischen Personen des Privatrechts. Sie hat keine Mitglieder, sondern allenfalls Destinatäre. Die Rechtsstellung der Begünstigten, der Destinatäre, wird im Wesentlichen durch die Satzung und den Stiftungszweck festgelegt. Nur im Rahmen des Stiftungszwecks sind Zuwendungen aus Mitteln der Stiftung zulässig. Fehlt insoweit eine Satzungsbestimmung, haben die Destinatäre keinerlei Mitgliedschaftsrechte und somit auch weder Verwaltungs- noch Kontrollbefugnisse. ${ }^{21}$ Die Stiftung nimmt durch ihren Vorstand am rechtsgeschäftlichen Verkehr teil. Der Vorstand ist an den in der Verfassung der Stiftung objektivierten Willen des Stifters gebunden. Die Stiftung muss über einen Vorstand im Sinne der $\S \S 86,26$ BGB verfügen. Der Vorstand ist das Organ, das die Stiftung im Rechtsverkehr vertritt. In der Regel wird der erste Vorstand im Stiftungsgeschäft bestellt. In der Satzung muss geregelt sein: die Größe des Vorstands, seine personelle Besetzung sowie das Bestellungs- und Abberufungsverfahren. Dabei reicht die Angabe einer Bandbreite, zum Beispiel zwei bis vier Personen, aus. ${ }^{22}$ Daneben können auch andere Organe wie ein Kuratorium, ein Stiftungs- oder ein Verwaltungsrat im Stiftungsgeschäft vorgesehen werden. In der Satzung sind bereits die Aufgaben und Kompetenzen der Or-

\footnotetext{
16 Fischer/Ihle (2008: 1694).

${ }^{17}$ Fischer/Ihle (2008: 1694); Schlüter/Stolte (2007: 59).

${ }^{18}$ Schlüter/Stolte (2007: 32, 59).

${ }^{19}$ Näher Fischer/Ihle (2008: 1694); Freundl (2004: 1510); Schlüter/Stolte (2007: 60).

${ }^{20}$ Schlüter/Stolte (2007: 61).

${ }^{21}$ Seifart/v. Campenhausen $\left({ }^{3} 2009\right.$ : $§ 8$ Rn. 132).

${ }^{22}$ Fischer/Ihle (2008: 1695 f.); Schlüter/Stolte (2007: 33, 58 f.).
} 
gane voneinander abzugrenzen. Im Gegensatz zu anderen Landesstiftungsgesetzen sieht das nordrhein-westfälische Landesstiftungsgesetz keine detaillierten Vorgaben hinsichtlich des Berufungsverfahrens, der Aufgaben und der Kompetenzen bei der Stiftungsorganisation vor, so dass Regelungen in der Satzung beziehungsweise in einer dieser als Anlage beizufügenden Geschäftsordnung vorgesehen werden können. ${ }^{23}$

\section{Stiftungssatzung: Privatautonomie und zivilrechtliche Mindestanforderungen}

Die Stiftung ist nach $\S 80$ Abs. 2 BGB als rechtsfähig anzuerkennen, wenn das Stiftungsgeschäft den Anforderungen des $\S 81$ Abs. 1 BGB genügt, die dauernde und nachhaltige Erfüllung des Stiftungszwecks gesichert erscheint und der Stiftungszweck das Gemeinwohl nicht gefährdet. Der Stifter hat privatautonom einen großen Spielraum, der nur durch die Gemeinwohlgefährdung eingeschränkt ist. ${ }^{24}$ In Hinblick auf Zweck, Kapital, Sitz, Name und Organisation der Stiftung genießt der Stifter die volle Privatautonomie. Er ist bei der Ausgestaltung der Satzung nicht an Formulierungsvorgaben gebunden, sondern hat Formulierungsfreiheit. ${ }^{25}$ Darum kann für jede Stiftung dem Zweck entsprechend eine maßgeschneidert passende Stiftungssatzung erstellt werden. Zivilrechtlich ist der Stifterwille also sehr weitreichend. Die Stiftungsverfassung wird allerdings limitiert durch zwingendes Bundes- und Landesrecht ( $\$ 85$ BGB). Dabei ist im Stiftungszivilrecht umstritten, ob einzelne Typen unternehmensverbundener Stiftungen zulässig sind. ${ }^{26}$

\section{Staatliches Anerkennungsverfahren und Reichweite der staatlichen Stiftungsaufsicht}

Die gesetzlichen Voraussetzungen, unter denen eine Stiftung Rechtsfähigkeit erlangt, werden im BGB einheitlich und abschließend bestimmt. ${ }^{27}$ Das Stiftungsgesetz für das Land Nordrhein-Westfalen ( $\$ 2$ StiftG NRW) verweist lediglich auf die Bestimmungen des BGB ( 80 Abs. 1 und 2 BGB). Rechtsfähige Stiftungen des privaten Rechts erlangen ihre Rechtsfähigkeit durch ihre Anerkennung seitens der zuständigen Landesbehörde $(\$ 80$ Abs. 1 BGB) ${ }^{28}$ In Nordrhein-Westfalen ist die Bezirksregierung Stiftungsaufsichtsbehörde. $^{29}$

Die Anerkennung ist nach $\S 80$ Abs. 2 BGB auszusprechen, wenn die in den $\S \S 80$ und 81 BGB abschließend bestimmten gesetzlichen Voraussetzungen vorliegen. Darum besteht ein Recht auf Stiftung. ${ }^{30}$ Im Stiftungszivilrecht geht es allein um das negative Tatbestandsmerkmal der Gemeinwohlgefährdung, das die privatautonome Stifterfreiheit ein-

\footnotetext{
${ }^{23}$ Fischer/Ihle (2008: 1695 f.).

${ }^{24}$ Werner ( ${ }^{12} 2008$ : § 81 Rn. 7).

${ }^{25}$ Heinrichs/Ellenberger ( ${ }^{68} 2009$ : $\S 81$ Rn. 5 ff.); Werner ( ${ }^{12} 2008: ~ § 81$ Rn. 7).

${ }^{26}$ Muscheler (2008a: $134 \mathrm{f}$ )); Muscheler (2008b: $179 \mathrm{f}$.).

27 Andrick (2005: 446).

${ }^{28}$ Hüttemann (2008: § 2 Rn. 54); Troll/Wallenhorst/Halaczinsky $\left({ }^{5} 2004\right.$ : A Rn. 38). Ob zwischen dem Zeitpunkt der Vornahme des Stiftungsgeschäfts und dem Tag der Anerkennung eine der Vorgesellschaft oder dem Vorverein vergleichbare Vorstiftung besteht, erscheint fraglich, weil kein Registrierungsverfahren vorgeschrieben ist (ablehnend Hüttemann 2008: $§ 2$ Rn. 54; anderer Ansicht Werner ${ }^{12} 2008$ : Vor $\S 80$ Rn. 22).

29 § 15 Abs. 2 StiftG NRW.

${ }^{30}$ Hüttemann (2008: § 2 Rn. 45).
} 
schränkt. ${ }^{31}$ Das Gemeinwohl ist gefährdet bei Verstoß gegen die Verfassung, ein allgemein gesetzliches Verbot oder gegen die guten Sitten. Das Erfordernis einer ,gemeinwohlkonformen Allzweckstiftung ${ }^{\text {‘32 }}$ ist darum nicht identisch mit den Gemeinwohlerfordernissen der Abgabenordnung (AO). Die Anerkennung durch die Stiftungsaufsichtsbehörde ist ein privatrechtsgestaltender Verwaltungsakt. ${ }^{33}$ In der Regel wird dem Stifter sowie dem im Stiftungsgeschäft benannten ersten Stiftungsvorstand eine Anerkennungsurkunde zugestellt. $^{34}$

Die Aufsicht über die durch Anerkennung rechtfähige Stiftung üben die Länder aus. Das BGB lässt nicht etwa offen, ob und gegebenenfalls in welchem Umfang die Länder eine staatliche Stiftungsaufsicht einrichten können. Es hat vielmehr die Entscheidung dem Grunde nach (abschließend) getroffen, dass der Staat das Leben sämtlicher von den $\S \S 80 \mathrm{ff}$. BGB erfassten Stiftungen flankiert und begleitet. Den Ländern überlässt es - auch über die Öffnungsklausel des § 85 BGB - die Befugnis, die Stiftungsaufsicht im Einzelnen zu gestalten, insbesondere Regelungen über die Zuständigkeit und den Umfang sowie die Intensität staatlicher Aufsichtsbefugnisse präventiver und repressiver Art zu treffen. ${ }^{35}$

Eine eingeschränkte Stiftungsaufsicht gilt bei privaten Stiftungen. Nach dem Stiftungsgesetz für das Land Nordrhein-Westfalen unterliegt nicht jeder Stiftungstyp der vollen staatlichen Aufsicht. Aus $§ 6$ Abs. 3 StiftG NRW folgt die Einschränkung, dass Stiftungen, die ausschließlich oder überwiegend private Zwecke verfolgen, nur insoweit der Stiftungsaufsicht unterliegen, als sicherzustellen ist, dass ihre Betätigung gesetzlich geschützten Interessen zuwiderläuft. Die Gesetzesbegründung rechtfertigt die abgesenkte staatliche Aufsicht damit, dass bei privatnützigen Stiftungen nicht in gleicher Weise wie bei gemeinnützigen Stiftungen eine staatliche Mitverantwortung für die Seriosität und kein gleichrangiges öffentliches Interesse daran bestehe, deren Ansehen und Vertrauen der Öffentlichkeit zu gewährleisten. ${ }^{36}$ Soweit die Stiftungen gemeinnützige Zwecke im Sinne der $\S \S 52 \mathrm{ff}$. AO verfolgen, sind sie nicht allein privatnützig, und darum unterliegen sie nicht nur der eingeschränkten, sondern der vollen Stiftungsaufsicht. ${ }^{37}$

In Nordrhein-Westfalen ist der Stiftungsaufsichtsbehörde ${ }^{38}$ durch den Stiftungsvorstand innerhalb von neun Monaten nach Ablauf des Geschäftsjahres eine Jahresabrechnung, eine Vermögensübersicht sowie ein Bericht über die Erfüllung des Stiftungszwecks vorzulegen ( $\$ 7$ Abs. 1 StiftG NRW). Diese Aufsichtsbehörde überwacht auch die Belastung oder Veräußerung erheblicher Vermögenswerte der Stiftung ( 7 Abs. 2 StiftG NRW) und beanstandet Maßnahmen der Stiftungsorgane, soweit sie dem Stiftungszweck zuwiderlaufen (§ 8 Abs. 1 StiftG NRW). Die Grundsätze ordnungsgemäßer Buchführung aus $§ 238$ HGB sind bei der Rechnungslegung in Nordrhein-Westfalen nur insoweit bindend, als die Stiftung durch einen unternehmerischen Geschäftsbetrieb die Kaufmannseigenschaft des $\S 1$ HGB erfüllt. ${ }^{39}$ Der vom Institut der Wirtschaftsprüfer (IDW) herausgegebene allgemeine

\footnotetext{
${ }^{31}$ Vgl. Schauhoff $\left({ }^{2} 2005\right.$ : $§ 3$ Rn. 20); Werner $\left({ }^{12} 2008: \S 80\right.$ Rn. 12).

${ }^{32}$ Wiederum Werner (122008: $\S 80$ Rn. 12).

33 Schlüter/Stolte (2007: 64).

${ }^{34}$ Schlüter/Stolte (2007: 65).

${ }^{35}$ Andrick (2005: 446).

${ }^{36}$ Gesetzesentwurf der Landesregierung, LT-Drucksache 13/5987, S. 14; dagegen kritisch Andrick (2005: 448).

${ }^{37}$ Andrick (2005: 448).

$38 § 15$ Abs. 2 StiftG NRW.

${ }^{39}$ Buchna ( ${ }^{9}$ 2008: 236); Troll/Wallenhorst/Halaczinsky ( ${ }^{5}$ 2004: B Rn. 11).
} 
Rechnungslegungsstandard für Stiftungen ${ }^{40}$ dient der Orientierung, ${ }^{41}$ er ist indes als Empfehlung eines Fachgremiums für Stiftungen nicht rechtlich bindend. Stets ist es Aufgabe der Rechnungslegung, einen Einblick in das vorhandene Vermögen sowie dessen weitere Erzielung und Verwendung zu gewährleisten. ${ }^{42}$

\section{Gemeinnützigkeitsrechtliche Vorgaben für Stiftungen - Satzungsmäßige Voraussetzungen}

Der Staat fördert private Stiftungen, die ihrerseits selbstlos und unmittelbar das Gemeinwohl fördern, durch weitreichende Steuerbefreiungen und -vergünstigungen $(\$ \S 51$ bis 68 $\mathrm{AO}$ in Verbindung mit den Einzelsteuergesetzen). ${ }^{43}$ Grund dafür sind, wie eingangs erwähnt, die Prinzipien der Staatssubstitution und der Subsidiarität. Bei der Errichtung und Verwaltung einer rechtsfähigen gemeinnützigen Stiftung sind neben den Vorschriften des Stiftungszivilrechts auch die steuerlichen Voraussetzungen des Gemeinnützigkeitsrechts zu beachten. Dazu gehört insbesondere das Erfordernis der satzungsmäßigen Gemeinnützigkeit ( $\$ 59$ und $60 \mathrm{AO}) .{ }^{44} \mathrm{Nach} \S 59 \mathrm{AO}$ wird die Steuervergünstigung nur gewährt, wenn sich

aus der Satzung, dem Stiftungsgeschäft oder der sonstigen Verfassung ergibt, welchen Zweck die Körperschaft verfolgt, dass dieser Zweck den Anforderungen der $\S \S 52$ bis 55 AO entspricht und dass er ausschließlich und unmittelbar verfolgt wird.

Dabei hat $§ 59$ AO eine doppelte Funktion: Einerseits ist er die gesetzliche Grundlage für den Grundsatz der satzungsmäßigen Gemeinnützigkeit und bestimmt andererseits den Mindestinhalt einer Satzung. ${ }^{45}$ Der steuerbegünstigte Zweck und die Art seiner Verwirklichung muss in der Satzung so genau bestimmt sein, dass allein aufgrund der Satzung geprüft werden kann, ob die Voraussetzungen für die Steuervergünstigung gegeben sind (\$60 AO). Seit dem Jahressteuergesetz 2009 muss die Satzung die in der dem Gesetz nunmehr als Anlage 1 beigefügten Mustersatzung bezeichneten Festlegungen enthalten. ${ }^{46}$ Zudem ist eine Vermögensbindungsklausel nach $§ 61$ Abs. 1 AO erforderlich. ${ }^{47}$

\section{Anforderungen an die tatsächliche Geschäftsführung der Stiftung}

Die Anforderungen an die tatsächliche Geschäftsführung der Stiftung ( $\$ 63$ Abs. $1 \mathrm{AO}$ ) sind in erster Linie die Selbstlosigkeit ( 55 AO), die Ausschließlichkeit (§ 56 AO) und die

\footnotetext{
${ }^{40}$ IDW-Stellungnahme zur Rechnungslegung: Rechnungslegung von Stiftungen (IDW RS HFA 5), WPg 2000, 391.

${ }^{41}$ Positiv Kußmaul/Meyering (2004: 376).

42 Schauhoff ( 2005 : $\$ 3$ Rn. 94).

43 Zum Überblick Seer/Versin (2007: 588).

${ }^{44}$ Schauhoff ( ${ }^{2} 2005: \$ 5$ Rn. 99).

${ }^{45}$ Hüttemann (2008: § 4 Rn. 122); Richter ( ${ }^{3} 2009$ : $\$ 98$ Rn. 122).

46 Art. 10 Nr. 14 Jahressteuergesetz 2009 v. 19.12.2008, BGB1. I 2008, 2794

${ }^{47}$ Hüttemann (2008: § 2 Rn. 59); vgl. auch Richter ( ${ }^{3} 2009$ : § 98 Rn. 128 ff.); Schauhoff ( ${ }^{2} 2005: \S 5$ Rn. 102) Durch das Jahressteuergesetz 2009 wurde die bislang durch $§ 62$ AO eröffnete Ausnahme von der satzungsmäBigen Vermögensbindung aufgehoben.
} 
Unmittelbarkeit der Zweckverwirklichung (§57 AO). Der Anwendungserlass zur Abgabenordnung (AEAO) konkretisiert die Anforderungen aus Verwaltungssicht. ${ }^{48}$

Selbstlosigkeit im Sinne des $§ 55$ AO meint die selbstlose, insbesondere nicht eigenwirtschaftliche Verfolgung der satzungsmäßigen Zwecke. ${ }^{49}$ Das Selbstlosigkeitsprinzip wird durch die Formulierung des $\S 55$ Abs. 1 Satz 1 AO „nicht in erster Linie“ eingeschränkt. Die Selbstlosigkeit ist aber erst dann nicht mehr gegeben, wenn die eigenwirtschaftlichen Interessen der Gesellschaft(er) das bestimmende Motiv des körperschaftlichen Wirkens darstellen. Allerdings kann auch eine gemeinnützige Stiftung in den Grenzen von $§ 58$ Nr. 5 AO einen Teil ihres Einkommens für die Versorgung des Stifters und seiner nächsten Angehörigen verwenden. Dies ist ein zulässiger Nebenzweck der gemeinnützigen Stiftung. 50

Ausschließlichkeit bedeutet, dass die gemeinnützige Stiftung ausschließlich ihre gemeinnützigen Zwecke verfolgen darf (§56 AO). Zwar schränken $§ 55$ Abs. 1 Satz 1 und 64 AO diesen Grundsatz ein, indem sie der Stiftung eigenwirtschaftliche Tätigkeit zum Zwecke der Mittelbeschaffung gestatten; dieser eigenwirtschaftliche Geschäftsbetrieb wird aber der Besteuerung unterworfen, so dass im Grunde kein Widerspruch zum Ausschließlichkeitsgrundsatz besteht. ${ }^{51}$

Die Stiftung ist grundsätzlich verpflichtet, ihre Zwecke unmittelbar zu verfolgen ( $\$ 57$ AO). Das bedeutet, dass das Tätigwerden der Stiftung darauf gerichtet sein muss, die steuerbegünstigten Zwecke direkt zu fördern, ohne dass weitere Umsetzungsaktivitäten dazwischengeschaltet sind oder sich die gemeinnützige Zweckerreichung lediglich als Folgewirkung der eigentlichen Stiftungsaktivitäten erweist. ${ }^{52}$ Eine mittelbare gemeinnützige Tätigkeit reicht grundsätzlich nicht aus. Eine Ausnahme stellt $§ 58$ Nr. 1 AO dar. Demnach kann eine Mittelbeschaffungskörperschaft, die sich darauf beschränkt, ihre Mittel überwiegend oder vollständig einer anderen, auch steuerbegünstigten Körperschaft zur Verwendung zu überlassen, gemeinnützig sein. ${ }^{53}$ Diese Tätigkeit als Mittelbeschaffungsstiftung empfiehlt sich für Stiftungen mit geringer Kapitalausstattung, weil geringe Verwaltungsressourcen ausreichen, um die Erträge nahezu ungeschmälert dem guten Zweck zuzuführen. ${ }^{54}$

Hinsichtlich der tatsächlichen Geschäftsführung treffen die gemeinnützige Stiftung spezielle Verfahrens- und Nachweispflichten. Die Gewährung gesetzlicher Steuervergünstigungen ist nicht an die Ordnungsmäßigkeit der Buchführung gekoppelt. Die Steuerverschonung bei steuerbegünstigten Körperschaften setzt aber ordnungsmäßige Aufzeichnungen im Sinne des $\S 63$ Abs. 3 AO voraus. Die Stiftung muss demnach mittels ordnungsgemäßer Aufzeichnungen über Einnahmen und Ausgaben den Nachweis führen, dass die tatsächliche Geschäftsführung auf ausschließliche und unmittelbare Erfüllung der steuerbegünstigten Zwecke gerichtet ist. Die handelsrechtlichen Rechnungslegungsvorschriften

\footnotetext{
${ }^{48}$ AEAO v. 2.1.2008, BStB1. I 2008, 26 (45 ff.); zum Teil neugefasst für das Gemeinnützigkeitsrecht durch AEAO v. 21.4.2008 BStB1. I 2008, 582.

${ }^{49}$ Richter ( ${ }^{3} 2009$ : $§ 98$ Rn. 36 ff.).

${ }^{50}$ Vgl. Bianchini-Hartmann/Richter (2008: 360 f.); Hüttemann (2008: $§ 2$ Rn. 60 und Rn. 48); Richter $\left({ }^{3} 2009\right.$ : $\S 98 \mathrm{Rn} .114)$.

51 Richter $\left({ }^{3} 2009\right.$ : $§ 98$ Rn. 72 ff.).

52 Fischer/Ihle (2008: 1697).

${ }^{53}$ Buchna ( ${ }^{9}$ 2008: 180 f.); Fischer/Ihle (2008: 1697); näher zur Überlassung finanzieller Mittel Troll/Wallenhorst/Halaczinsky ( ${ }^{5}$ 2004: C Rn. $111 \mathrm{ff}$.).

54 Vgl. Fischer/Ihle (2008: 1697).
} 
sind nur insoweit zu beachten, als sich dies aus der Rechtsform oder wirtschaftlichen Tätigkeit ergibt. ${ }^{55}$

\section{Stiftungsrechtliches Gebot der Kapitalerhaltung und stiftungssteuerrechtliches Gebot zeitnaher Mittelverwendung}

Beim Vermögenseinsatz gemeinnütziger Stiftungen sind Verbindungslinien von Stiftungszivilrecht und Stiftungssteuerrecht zu beachten: Nach dem dispositiven ${ }^{56}$ Grundsatz der Kapitalerhaltung darf das Stiftungskapital nicht angegriffen werden und die Stiftung darf ihre Förderung lediglich aus den Erträgen erbringen ( 4 StiftG NRW). ${ }^{57}$ Die stiftungszivilrechtlichen Möglichkeiten und die Vorgaben des steuerlichen Gemeinnützigkeitsrechts stehen dabei nicht unverbunden nebeneinander. Nach zutreffender Ansicht werden die gemeinnützigkeitsrechtlichen Vorgaben insbesondere zur Vermögensverwendung $(\$ \S 55,58$ AO) nicht durch das stiftungsrechtliche Prinzip der Vermögenserhaltung verdrängt. ${ }^{58}$ Vielmehr ist es genau umgekehrt: Durch das Erfordernis der satzungsmäßigen Gemeinnützigkeit werden die Stiftungsvorstände auch mit den Mitteln des Stiftungsrechts zur Erhaltung der steuerlichen Rahmenbedingungen verpflichtet. Eine steuerlich unzulässige Rücklagenbildung ist daher auch von den Stiftungsbehörden als satzungswidrig zu beanstanden. Daher müssen sich die Vermögenserhaltungsstrategien der Stiftung an den Vorgaben der $\S \S$ 55 Abs. 1 Nr. 5, 58 Nr. 6, Nr. 7, Nr. 11 und Nr. 12 AO orientieren. ${ }^{59}$ Dies illustriert, dass bereits mit der Errichtung der Satzung die Weichen zwischen der privatnützigen und der gemeinnützigen Stiftung gestellt werden. Bei der gemeinnützigen Stiftung werden bereits in der Satzung planmäßig beide Rechtsschichten durch den Stifter miteinander verbunden.

Bei der tatsächlichen Geschäftsführung müssen die Restriktionen des Gemeinnützigkeitsrechts beachtet werden: So ist die zeitnahe Mittelverwendung Aufgabe und zugleich Bürde der gemeinnützigen Stiftung. ${ }^{60} \S 55$ Abs. 1 Nr. 5 AO normiert den Grundsatz der zeitnahen Mittelverwendung. Eine steuerbegünstigte Stiftung muss die ihr in einem Geschäftsjahr zugeflossenen Mittel bis zum Ende des folgenden Jahres für ihre Satzungszwecke verwandt haben. Verwendung ist dabei auch die Anschaffung oder Herstellung von Vermögensgegenständen, die satzungsmäßigen Zwecken dient. Am Ende des Kalender- oder Wirtschaftsjahres noch vorhandene Mittel müssen in der Bilanz oder der Vermögensaufstellung dem Vermögen oder einer zulässigen Rücklage zugeordnet werden. Sind Mittel für die Verwendung in Folgejahren bestimmt, ist dies durch eine eigenständige Mittelverwendungsrechnung nachzuweisen. ${ }^{61}$ Das Vermögen der Stiftung, auch soweit es durch Umschichtungen entstanden ist, unterliegt nicht dem Gebot der zeitnahen Mit-

\footnotetext{
55 Kußmaul/Meyering (2004: 371) mit weiteren Nachweisen.

${ }^{56}$ Soweit nicht in der Satzung etwas anderes bestimmt ist oder der Wille der Stifterin oder des Stifters auf andere Weise nicht verwirklicht werden kann, ist das Stiftungsvermögen ungeschmälert zu erhalten (§4 Abs. 2 Satz 1 StiftG NRW); näher zur so genannten Verbrauchsstiftung Werner (122008: Vor $\S 80$ Rn. 24) mit weiteren Nachweisen.

${ }^{57}$ Näher Schlüter/Stolte (2007: 113 ff.).

58 Überzeugend Hüttemann (2008: § 2 Rn. 60) mit weiteren Nachweisen; ebenso Neuhoff (122000: Vor $§ 80$ Rn. $92 \mathrm{f}$.).

${ }^{59}$ Vgl. Hüttemann (2008: $§ 2$ Rn. 60 und Rn. 48).

${ }^{60}$ Thiel (1992: 1900).

${ }^{61}$ AEAO Nr. 27 zu $\$ 55$ AO; Beispiele für eine Mittelverwendungsrechnung bei Buchna $\left({ }^{9} 2008: 125\right)$ sowie Richter ( ${ }^{3} 2009$ : $\$ 98$ Rn. 80).
} 
telverwendung. ${ }^{62}$ Mittelverwendung für satzungswidrige Zwecke ist der Stiftung freilich untersagt ( $\$ 55$ Abs. 1 Nr. 1 und 3 AO).

\section{Überprüfung der Gemeinnützigkeitsanforderungen durch die Finanzbehörde}

Ein stiftungssteuerliches „Anerkennungsverfahren“ parallel zum stiftungszivilrechtlichen gibt es nicht. Auch ist ein besonderes Prüfungsverfahren für die steuerliche Gemeinnützigkeit gesetzlich nicht vorgesehen. ${ }^{63}$ Vielmehr prüft die Finanzverwaltung die Voraussetzungen der Gemeinnützigkeit im laufenden Veranlagungs- und Festsetzungsverfahren. ${ }^{64}$ Dies ist dem Umstand geschuldet, dass neben den satzungsmäßigen Voraussetzungen auch die tatsächliche Geschäftsführung den Vorschriften des Gemeinnützigkeitsrechts entsprechen muss ( 63 Abs. 1 AO). Diese Voraussetzung kann die Finanzverwaltung aber logischerweise erst im Nachhinein überprüfen. Das zuständige Finanzamt kann allerdings von der jährlichen Anforderung zur Abgabe von Steuererklärungen absehen. Dies entspricht einer verbreiteten Praxis. Soweit keine wirtschaftlichen zu besteuernden Tätigkeiten ausgeübt werden, wird demnach ein Freistellungsbescheid für die Dauer von drei Jahren erteilt. ${ }^{65}$ Das Finanzamt kann auf Antrag der Stiftung zunächst eine vorläufige Bescheinigung der Gemeinnützigkeit erteilen. ${ }^{66}$

\section{Fazit: Das Ziel der maßgeschneiderten Stiftungssatzung}

Die Privatautonomie gewährt dem Stifter große Freiheiten. Bei der gemeinnützigen Stiftung sind indes die angerissenen Vorgaben der Abgabenordnung bereits bei der Errichtung der Satzung zu beachten. Der ungeschulte Laie kann in der Regel nur mit Hilfe fachlicher juristischer Unterstützung durch die Anerkennungsbehörde oder Rechtsberatung eine wirksame durchführbare Stiftungssatzung festlegen. ${ }^{67}$ Zwischen einer bloß wirksamen und einer maßgeschneiderten Satzung liegen indes Welten. Daher ist dem Stifter eine rechtliche und steuerrechtliche Beratung dringend anzuraten.

Auf dem Weg in die steuerliche Gemeinnützigkeit ist dem Stifter und seinen Beratern ohnehin zu empfehlen, vor der Gründung die Satzung dem zuständigen Finanzamt zur Prüfung vorzulegen. ${ }^{68}$ Denn einerseits können auf diesem Wege präventiv bereits Formulierungen abgestimmt und Satzungsmängel vermieden werden. Zum anderen gewährt die Finanzverwaltung für geprüfte Satzungen einen Vertrauensschutz. Dabei bleiben Satzungsverstöße folgenlos. ${ }^{69}$ Gerade mit Blick auf den Vertrauensschutz prüfen die Finanzämter die Satzung neu errichteter Körperschaften intensiver. ${ }^{70}$ Es gilt also der Rat, in

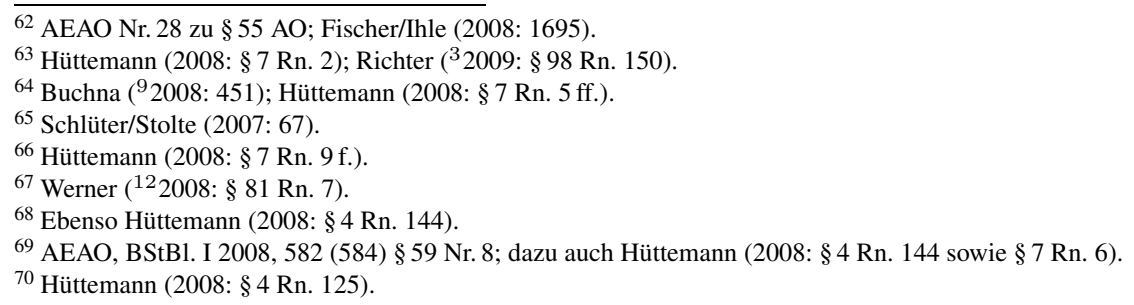


enger Kooperation mit dem zuständigen Finanzamt den Weg in die Gemeinnützigkeit einzuschlagen.

Wem der aufgezeigte Weg zu einer eigenen rechtsfähigen gemeinnützigen Stiftung zu steinig erscheint, weil das für die gute Tat reservierte Vermögen diese Hürden nicht zu überwinden vermag, für den gibt es aber auch eine Zustiftung als unsteinigen Weg. Bei der bloßen Zustiftung wird keine selbständige Körperschaft gegründet. ${ }^{71}$ Auch aus steuerrechtlicher Sicht gibt es keinen Zwang für die Errichtung einer eigenständigen Stiftung, weil auch Zustiftungen nunmehr begünstigt sind. ${ }^{72}$

\section{Literatur}

ANDRICK, Berndt (2005). „Das Stiftungsgesetz für das Land Nordrhein-Westfalen“, NordrheinWestfälische Verwaltungsblätter, 445-450.

Bianchini-Hartmann, Maren und Andreas Richter (2008). „Die Besteuerung von Familienstiftungen“, in: Dieter BIRK (Hrsg.). Transaktionen Vermögen Pro Bono, Festschrift zum zehnjährigen Bestehen von P+P Pöllath + Partners. München, 337-362.

BuCHNA, Johannes $\left({ }^{9} 2008\right)$. Gemeinnützigkeit im Steuerrecht. Achim.

DRÜEN, Klaus-Dieter (2008). ,Steuerliche Förderung von Wissenschaft und Forschung“, in: Alfons LABISCH (Hrsg.). Jahrbuch der Heinrich-Heine-Universität Düsseldorf 2007/2008. Düsseldorf, 443-455.

FISCHER, Daniel und Jörg IHLE (2008). „Satzungsgestaltung bei gemeinnützigen Stiftungen“, Deutsches Steuerrecht, 1692-1698.

FÖRSTER, Guido (2009). „Steuerliche Rahmenbedingungen für Stiftungsmaßnahmen“, in: H. Michael PIPER (Hrsg.). Jahrbuch der Heinrich-Heine-Universität Düsseldorf 2008/2009. Düsseldorf, 677-687.

FREUNDL, Fabian (2004). „Die Stiftung - das Gestaltungsinstrument der Unternehmensnachfolge“, Deutsches Steuerrecht, 1509-1514.

HEINICKE, Wolfgang $\left({ }^{28} 2009\right)$, in: Ludwig SCHMIDT. Einkommensteuergesetz Kommentar. München.

HeinRICHS, Helmut und Jürgen ElLENBERGER $\left({ }^{68} 2009\right)$, in: Otto PALANDT. Bürgerliches Gesetzbuch. München.

HÜtTEMAnN, Rainer (2008). Gemeinnützigkeits- und Spendenrecht. Köln.

Kussmaul, Heinz und Stephan Meyering (2004). „Die Rechnungslegung der Stiftung“, Deutsches Steuerrecht, 371-376.

MUSCHELER, Karheinz (2008a). „Die Unternehmensstiftung, besser: Die unternehmensverbundene Stiftung, Teil 1“, Zeitschrift für die gesammte erbrechtliche Praxis, 134-143.

MUSCHELER, Karheinz (2008b). „Die Unternehmensstiftung, besser: Die unternehmensverbundene Stiftung, Teil 2“, Zeitschrift für die gesammte erbrechtliche Praxis, 174-181.

NeUHOFF, Klaus $\left({ }^{12} 2000\right)$, in: Hans Theodor SOER GEL. Bürgerliches Gesetzbuch, $\S \S 1-103$. Frankfurt am Main.

RICHTER, Andreas ( $\left.{ }^{3} 2009\right)$. Handbuch des Gesellschaftsrechts. Bd. 5. München.

SCHAuhofF, Stephan $\left({ }^{2} 2005\right)$. Handbuch der Gemeinnützigkeit. München.

SCHLÜTER, Andreas und Stefan STOLTE (2007). Stiftungsrecht. München.

\footnotetext{
${ }^{71}$ Eingehend zur Zustiftung Werner (2003).

${ }^{72}$ Seit dem Jahr 2007 sind Spenden in den Vermögensstock durch einen erhöhten Spendenabzug nach $\$ 10$ Abs. 1a EStG auch dann begünstigt, wenn keine neue Stiftung begründet wird, sondern die Gründung einer bloßen Zustiftung erfolgt. Dazu näher der folgende Beitrag von Förster.
} 
SEER, Roman (2003). „Gemeinwohlzwecke und steuerliche Entlastung“, in: Monika JACHMANN (Hrsg.). Gemeinnützigkeit, Deutsche Steuerjuristische Gesellschaft. Bd. 26. Köln, 11-48.

SEER, Roman und Volker VERSIN (2007). „Die gemeinnützige Stiftung im Steuerrecht“, Steuer und Studium, 588-594.

SeIFART, Werner und Axel von CAMPENHAUSEN ( $\left.{ }^{3} 2009\right)$. Stiftungsrechts-Handbuch. München.

THIEL, Jens (1992). „Die zeitnahe Mittelverwendung - Aufgabe und Bürde gemeinnütziger Körperschaften“, Der Betrieb, 1900-1907.

Troll, Max, Rolf Wallenhorst und Raymond HalacZinsky $\left({ }^{5} 2004\right)$. Die Besteuerung gemeinnütziger Vereine, Stiftungen und der juristischen Personen des öffentlichen Rechts. München.

WERnER, Almuth (2003). Die Zustiftung. Baden-Baden.

WERNER, Rüdiger (2006). „Stiftungen als Instrument der Unternehmens- und Vermögensnachfolge“, Zeitschrift für Erbrecht und Vermögensnachfolge, 539-644.

WERNER, Olaf ( $\left.{ }^{12} 2008\right)$, in: Walter ERMAN. Bürgerliches Gesetzbuch. Bd. 1, § 80. Köln. 
